\title{
Factors that predict 30-day readmission after bariatric surgery: experience of a publicly funded Canadian centre
}

\author{
Jerry T. Dang, ${ }^{*} \mathrm{MD}$ \\ Iran Tavakoli,* MSc, MD \\ Noah Switzer, MPH, MD \\ Valentin Mocanu, MD \\ Xinzhe Shi, MPH \\ Chris de Gara, MB, MS \\ Daniel W. Birch, MSc, MD \\ Shahzeer Karmali, MPH, MD
}

${ }^{*}$ Contributed equally as co-first authors.

Accepted Aug. 30, 2019

\author{
Correspondence to: \\ J. Dang \\ Department of Surgery \\ University of Alberta Hospital \\ 8440112 St NW \\ Edmonton AB T6G 2B7 \\ dang2@ualberta.ca
}

DOI: $10.1503 /$ cjs.014918
Background: Hospital readmissions after bariatric surgery can significantly increase health care costs. Rates of readmission after bariatric surgery have ranged from $0.6 \%$ to $11.3 \%$, but the rate of complications and the factors that predict readmission have not been well characterized in Canada. The objective of this study was to characterize readmission rates and the factors that predict 30-day readmission in a Canadian centre.

Methods: A retrospective study was performed on all patients who underwent bariatric surgery between 2010 and 2015 in a single Canadian centre. Procedures included laparoscopic Roux-en-Y gastric bypass (LRYGB), laparoscopic sleeve gastrectomy (LSG) and laparoscopic adjustable gastric banding (LAGB). Prospectively collected data were extracted from an administrative database. Multivariable logistic regression analysis was performed to determine which factors predict 30-day readmission.

Results: A total of 1468 patients had bariatric surgery (51.0\% LRYGB, 40.5\% LSG, $8.6 \%$ LAGB) during the 6-year study period, with an overall 30-day readmission rate of $7.5 \%$. LRYGB was associated with a higher readmission rate $(11.4 \%)$ than LSG $(3.7 \%)$ or LAGB (1.6\%). Common reasons for readmission were infection $(24.8 \%)$, pain $(17.4 \%)$ and nausea or vomiting (10.1\%). Multivariable analysis identified 3 factors that independently predicted readmission: length of stay greater than 4 days (odds ratio [OR] 2.18, $95 \%$ confidence interval $[\mathrm{CI}] 1.03-4.63, p=0.042)$, LRYGB (OR $5.21,95 \% \mathrm{CI}$ $1.19-22.73, p=0.028$ ) and acute renal failure (OR 14.10, 95\% CI 1.07-186.29, $p=0.045$ ).

Conclusion: Readmissions after bariatric surgery were most commonly caused by potentially preventable factors, such as pain, nausea or vomiting. Strategies to identify and address factors associated with readmission may reduce readmissions and health care costs after bariatric surgery in a publicly funded health care system.

Contexte : Les réadmissions hospitalières après la chirurgie bariatrique peuvent accroître significativement le coût des soins de santé. Les taux de ces réadmissions ont varié de $0,6 \%$ à $11,3 \%$, mais le taux de complications et les facteurs de prédiction des réadmissions n'ont pas été bien caractérisés au Canada. L'objectif de cette étude est de caractériser les taux de réadmissions et les facteurs qui permettent de prédire une réadmission à 30 jours dans un centre canadien.

Méthodes : Nous avons étudié rétrospectivement tous les cas de chirurgie bariatrique effectués entre 2010 et 2015 dans un établissement canadien. Les interventions incluaient la dérivation gastrique Roux-en-Y laparoscopique (DGRYL), la gastrectomie en manchon laparoscopique (GML) et la gastroplastie laparoscopique avec anneau ajustable (GLAA). Les données recueillies de manière prospective ont été extraites d'une base de données administrative. Nous avons procédé à une analyse de régression logistique multivariée pour déterminer quels facteurs permettaient de prédire la réadmission à 30 jours.

Résultats : En tout, 1468 patients ont subi une chirurgie bariatrique (51,0\% DGRYL, 40,5\% GML et 8,6\% GLAA) durant les 6 années de l'étude, avec un taux global de réadmission à 30 jours de $7,5 \%$. La DGRYL a été associée un taux de réadmission plus élevé $(11,4 \%)$ que la GML $(3,7 \%)$ ou la GLAA $(1,6 \%)$. Les raisons de réadmission les plus fréquentes ont été infection $(24,8 \%)$, douleur $(17,4 \%)$ et nausées ou vomissements $(10,1 \%)$. L'analyse multivariée a permis de dégager 3 facteurs indépendants de réadmission, soit séjour de plus de 4 jours (rapport des cotes [RR] 2,18, intervalle de confiance [IC] de 95\% 1,03-4,63, $p=$ $0,042$ ), DGRYL (RC 5,21, IC de 95\% 1,19-22,73, $p=0,028)$ et insuffisance rénale aiguë (RC 14,10, IC de $95 \%$ 1,07-186,29, $p=0,045$ ).

Conclusion : Les réadmissions après la chirurgie bariatrique ont le plus souvent été dues à des facteurs évitables, tels que douleur, nausées et vomissements. Des stratégies visant à identifier et à corriger les facteurs de réadmission pourraient réduire ces dernières et le coût des soins de santé après la chirurgie bariatrique dans un système de santé public. 
0 besity has become a global epidemic. ${ }^{1}$ The prevalence of obesity in Canada is nearing 30\%, ${ }^{2}$ and the health care costs associated with obesity in this country are now estimated to be between Can $\$ 4.6$ billion and Can\$7.1 billion. ${ }^{3}$ In the United States alone, the cost of treating obesity and its complications is approaching US\$200 billion per year. ${ }^{4}$ At present, bariatric surgery is the only effective way for patients with obesity to achieve significant long-term weight loss, and it associated with substantial improvement in obesity-related comorbidities. $^{5-7}$ As a result, the number of bariatric surgeries performed worldwide has increased substantially over the last decade. $^{8}$

As the popularity of bariatric surgery has increased, questions regarding the safety of the procedures have been raised. Although many studies have demonstrated that bariatric procedures are safe, a small proportion of patients experience at least 1 major adverse event within the first 30 days after surgery. Adverse events also result in costly hospital readmissions. In Canada, the largest bariatric collaborative from Ontario reported an overall complication rate of $11.7 \%$ in a study of 5000 procedures. ${ }^{9}$ Studies investigating safety outcomes and utilization of resources 6 months after bariatric surgery also report that such complications result in costly readmissions and emergency department visits. ${ }^{10}$

Readmission rates have ranged between $0.6 \%$ and $11.3 \%$ depending on the procedure type in different cohorts of patients undergoing bariatric surgery. ${ }^{6,11-13}$ Hospital readmissions increase the average cost of a bariatric operation from approximately \$US27 000 to \$US65 000. ${ }^{10,14}$ In 2008, the National Quality Forum in the US identified hospital readmission as a central factor in the evaluation of hospital performance. Studying factors that predict readmission is a potential strategy to identify ways to substantially reduce costs in a publicly funded health care system with scarce resources.

In the present study we examined the trends and predictors of readmission in a high-volume accredited Canadian bariatric centre from 2010 to 2015 . Our primary aim was to characterize hospital readmission rates and their causes within 30 days of surgery. Our secondary aim was to identify independent predictors of readmission.

\section{Methods}

\section{Study design}

In this retrospective cohort study we identified all patients who underwent primary bariatric surgery between January 2010 and December 2015 at the Edmonton Adult Bariatric Specialty Clinic, which is a single, high-volume, publicly funded bariatric surgery centre in Edmonton, Alberta. This centre is the only dedicated bariatric surgical program in the province of
Alberta. It has a complement of 4 full-time bariatric surgeons performing a full array of bariatric procedures. Nearly 1000 patients are assessed each year at the centre, and approximately 250 primary bariatric surgery procedures are performed per year. At the centre, patients are initially assessed and treated by specialized bariatric physicians along with nurses, dieticians, physiotherapists and psychologists. The bariatric surgeon offers either laparoscopic Roux-en-Y gastric bypass (LRYGB) or laparoscopic sleeve gastrectomy (LSG) depending on patient preference unless there are contraindications to a specific procedure. Laparoscopic adjustable gastric banding (LAGB) is also available but has been infrequently performed at the centre since 2014 because of high rates of weight loss failure and long-term complications. ${ }^{15}$ Postoperatively, all patients undergo an upper gastrointestinal contrast study to assess for leaks on postoperative day 1 . Patients are typically discharged on postoperative day 1 or 2 after achieving the following discharge criteria: normal vital signs, adequate pain control, tolerating a full fluid diet, ambulating well and being agreeable to discharge. Patients are then seen for follow-up in clinic in 3 to 4 weeks. Ethics approval for this research was obtained from the Health Research Ethics Board of the University of Alberta. The requirement for informed consent was waiaved because of the retrospective nature of the study.

\section{Inclusion and exclusion criteria}

All patients who underwent bariatric surgery between January 2010 and December 2015 were included. The surgical procedures performed were primarily LRYGB and LSG. Patients who had undergone bariatric surgery previously were excluded.

\section{Data extraction}

The Data Integration, Measurement and Reporting (DIMR) database is an administrative database that prospectively collects data for all hospital admissions in Edmonton. Data on patient demographic characteristics, surgical procedures, length of stay, operating time, perioperative complications and readmissions were collected for the study population. These were coded using the International Statistical Classification of Diseases and Related Health Problems, 10th Revision (ICD-10-CA). Comorbidity and morbidity data were derived from diagnostic and procedural codes. It is important to note that body mass index (BMI) is not captured by this database and was not included in the analysis.

For this study, DIMR extracted and linked data from Alberta Health Services clinical systems with data from the Discharge Abstract Database. Data from the Discharge Abstract Database included data extracted as ICD-10-CA codes, and data from the clinical systems included date of 
procedure, type of procedure, operative time, length of stay, sex, and date of readmission. The main outcome of interest was hospital readmission within 30 days of surgery. We also aimed to assess factors that predicted readmission.

\section{Definitions of variables}

Anastomotic leak did not have a specific ICD-10-CA code and was defined as an operation with washout but without hemorrhage that included the following codes: 1OT52DA, 1OT52DATS, 1OT52LA and 1OT52LATS. This interpretation was based on a previous study using ICD-10-CA codes. ${ }^{9}$ Reoperation was defined as an unplanned operation during the initial admission. Major complications were defined as a composite variable including any complication that occurred during the initial admission. Major complications included any of the following: bleeding, infection, respiratory failure, bowel obstruction, renal failure, cardiac arrest, myocardial infarction or fascial dehiscence.

\section{Statistical analysis}

Statistical analysis was performed using Stata 14. Continuous variables were reported as means and standard deviations and categorical variables were summarized by percentages. Preoperative factors identified in this analysis included the following: age, sex, hypertension, diabetes, chronic obstructive pulmonary disease, coronary artery disease, chronic kidney disease, hyperlipidemia, gastroesophageal reflux disease, obstructive sleep apnea, asthma, osteoarthritis and smoking. Perioperative factors included procedure type, operative time and length of stay. Postoperative complications included leak, bleeding, infections, reoperation, hypotension, intestinal obstruction, air embolism, dehiscence, delirium, renal failure, respiratory failure, venous thromboembolism and cardiac events.

Univariate and multivariable logistic regression was performed to determine perioperative factors that were statistically associated with 30-day readmission. We used a purposeful selection method to develop our logistic regression model. Any variable with a $p$ value less than 0.2 in the univariate analysis was included in the multivariable analysis. In the multivariable model, significant variables (Wald test $p<$ $0.05)$ were identified. Important variables that were removed were assessed for confounding effects. If the change in the regression coefficients of other variables was greater than $15 \%$, then the variable remained in the model. Linear assumption of continuous variables and collinearity were checked. The Cook distance test was used to detect outliers and influential observations in the predictive model. The Hosmer-Lemeshow test was used to test the goodness of fit for the model.

\section{Results}

A total of 1468 patients had primary bariatric surgery from 2010 to 2015 . The majority of patients underwent LRYGB (51.0\%), followed by LSG (40.5\%) and LAGB (8.6\%). The basic demographic characteristics of patients who were readmitted and those who were not are summarized in Table 1. These characteristics are summarized for each procedure type in Table 2. Overall, the mean age of patients was 44.7 (standard deviation 10.1) years, and $82.4 \%$ of the patients were female. The most common comorbidities were hypertension (55.3\% of all patients), type 2 diabetes $(37.6 \%)$ and obstructive sleep apnea (34.8\%) (Table 1).

The overall 30 -day readmission rate was $7.5 \%$, with rates varying between $3.0 \%$ and $9.7 \%$ over the study period (Table 3). Patients who underwent LRYGB had the highest readmission rate (11.4\%), followed by patients who underwent LSG (3.7\%) and LAGB (1.6\%). There was 1 death within 30 days of surgery over the 6 years of our study. The mean length of stay for readmissions was 4.8 days, and $70 \%$ of patients who were readmitted remained in hospital for 4 or fewer days. The majority of patients who were readmitted $(66.1 \%)$ were readmitted early, within the first 10 days after discharge. The main

\begin{tabular}{|c|c|c|c|c|}
\hline \multirow[b]{2}{*}{ Characteristic } & \multicolumn{3}{|c|}{ No. (\%) of patients; ${ }^{*}$ readmission status } & \multirow[b]{2}{*}{$p$ value } \\
\hline & $\begin{array}{c}\text { Total } \\
n=1468\end{array}$ & $\begin{array}{c}\text { Readmitted } \\
n=109\end{array}$ & $\begin{array}{c}\text { Not readmitted } \\
n=1358\end{array}$ & \\
\hline Age, yr, mean \pm SD & $44.7 \pm 10.1$ & $44.0 \pm 10.2$ & $44.8 \pm 10.1$ & 0.42 \\
\hline Female & 1209 (82.4) & $94(86.2)$ & $1115(82.1)$ & 0.27 \\
\hline \multicolumn{5}{|l|}{ Preoperative comorbidity } \\
\hline Hypertension & $811(55.3)$ & $70(64.2)$ & $741(54.5)$ & 0.050 \\
\hline Type 2 diabetes & $552(37.6)$ & $42(38.5)$ & $510(37.5)$ & 0.84 \\
\hline Osteoarthritis & $61(4.2)$ & $4(3.7)$ & $57(4.2)$ & 0.79 \\
\hline Coronary artery disease & $13(0.9)$ & $2(1.8)$ & $11(0.8)$ & 0.27 \\
\hline Chronic kidney disease & $12(0.8)$ & $1(0.9)$ & $11(0.8)$ & 0.90 \\
\hline Hyperlipidemia & $137(9.3)$ & $7(6.4)$ & $130(9.6)$ & 0.28 \\
\hline GERD & $245(16.7)$ & $17(15.6)$ & $228(16.8)$ & 0.75 \\
\hline Obstructive sleep apnea & $511(34.8)$ & 38 (34.9) & $473(34.8)$ & 0.99 \\
\hline COPD & $19(1.3)$ & $2(1.8)$ & $17(1.3)$ & 0.60 \\
\hline Asthma & $182(12.4)$ & 15 (13.8) & 167 (12.3) & 0.65 \\
\hline
\end{tabular}


diagnoses for readmission were infectious complications (24.8\%), pain (17.4\%) and nausea or vomiting (10.1\%) (Fig. 1).

Univariate analysis identified the following significant variables for inclusion in the multivariable model $(p<0.20)$ : hypertension, procedure type, operative time, length of stay, cardiac events, infections, renal failure, respiratory failure, hypotension, delirium, leak, reoperation and wound dehiscence (Table 1 and Table 4). When we checked for linearity of continuous variables, length of stay was not linear and was separated into categories. No clinically significant interactions were found. The variables included in the final model are listed in Table 5.

Overall, multivariable logistic regression analysis identified 3 independent predictors of hospital readmission at 30 days: initial length of stay longer than 4 days, LRYGB procedure and the development of acute renal failure during the initial hospital admission (Table 5). The overall rate of major complications was $10.2 \%$; these complications were mostly bleeding (4.2\%), infections (3.8\%) and cardiac events (2.3\%) (Table 4).

\section{Discussion}

This study is one of the largest analyses of readmissions following bariatric surgery in a publicly funded health care system. In this retrospective review of prospectively collected data, we report a readmission rate of $7.5 \%$ after bariatric surgery in a single large-volume Canadian centre. This is similar to rates reported for various bariatric cohorts across the US and Canada. ${ }^{13,16-18}$ Readmission rates vary between studies largely because of differences in the type of procedure predominantly performed. For example, LAGB is typically associated with lower readmission and early complication rates, but in our study only
Table 2. Patient demographic characteristics and preoperative comorbidities by procedure

\begin{tabular}{|c|c|c|c|c|}
\hline \multirow[b]{2}{*}{ Characteristic } & \multicolumn{3}{|c|}{ No. $(\%)$ of patients; ${ }^{*}$ procedure } & \multirow[b]{2}{*}{$p$ value } \\
\hline & $\begin{array}{l}\text { LRYGB } \\
n=748\end{array}$ & $\begin{array}{c}\text { LSG } \\
n=594\end{array}$ & $\begin{array}{c}\text { LAGB } \\
n=126\end{array}$ & \\
\hline Age, yr, mean $\pm S D$ & $44.6 \pm 9.6$ & $45.0 \pm 10.4$ & $44.7 \pm 11.3$ & 0.95 \\
\hline Female & $607(81.2)$ & 495 (83.3) & $107(84.9)$ & 0.42 \\
\hline Hypertension & $443(59.2)$ & $299(50.3)$ & $69(54.8)$ & 0.005 \\
\hline Diabetes & $320(42.8)$ & $185(31.1)$ & 47 (37.3) & $<0.001$ \\
\hline Osteoarthritis & $36(4.8)$ & $21(3.5)$ & $4(3.2)$ & 0.43 \\
\hline Coronary artery disease & $9(1.2)$ & $2(0.3)$ & $2(1.6)$ & 0.16 \\
\hline Chronic kidney disease & $7(0.9)$ & $3(0.5)$ & $2(1.6)$ & 0.41 \\
\hline Hyperlipidemia & $75(10.0)$ & $47(7.9)$ & $15(11.9)$ & 0.24 \\
\hline GERD & $121(16.2)$ & $102(17.2)$ & $22(17.5)$ & 0.86 \\
\hline Obstructive sleep apnea & $273(36.5)$ & $206(34.7)$ & $32(25.4)$ & 0.05 \\
\hline COPD & $15(2.0)$ & $4(0.7)$ & $0(0.0)$ & 0.041 \\
\hline Asthma & $95(12.7)$ & 76 (12.8) & $11(8.7)$ & 0.42 \\
\hline
\end{tabular}

Table 3. Patients readmitted in each study year

\begin{tabular}{|ccc|}
\hline Year & $\begin{array}{c}\text { Total } \\
\text { no. of patients }\end{array}$ & $\begin{array}{c}\text { No. (\%) of readmitted } \\
\text { patients }\end{array}$ \\
\hline 2010 & 189 & $13(6.9)$ \\
\hline 2011 & 197 & $6(3.0)$ \\
\hline 2012 & 250 & $18(7.2)$ \\
\hline 2013 & 278 & $19(6.8)$ \\
\hline 2014 & 277 & $27(9.7)$ \\
\hline 2015 & 277 & $27(9.7)$ \\
\hline Total & 1468 & $110(7.5)$ \\
\hline
\end{tabular}

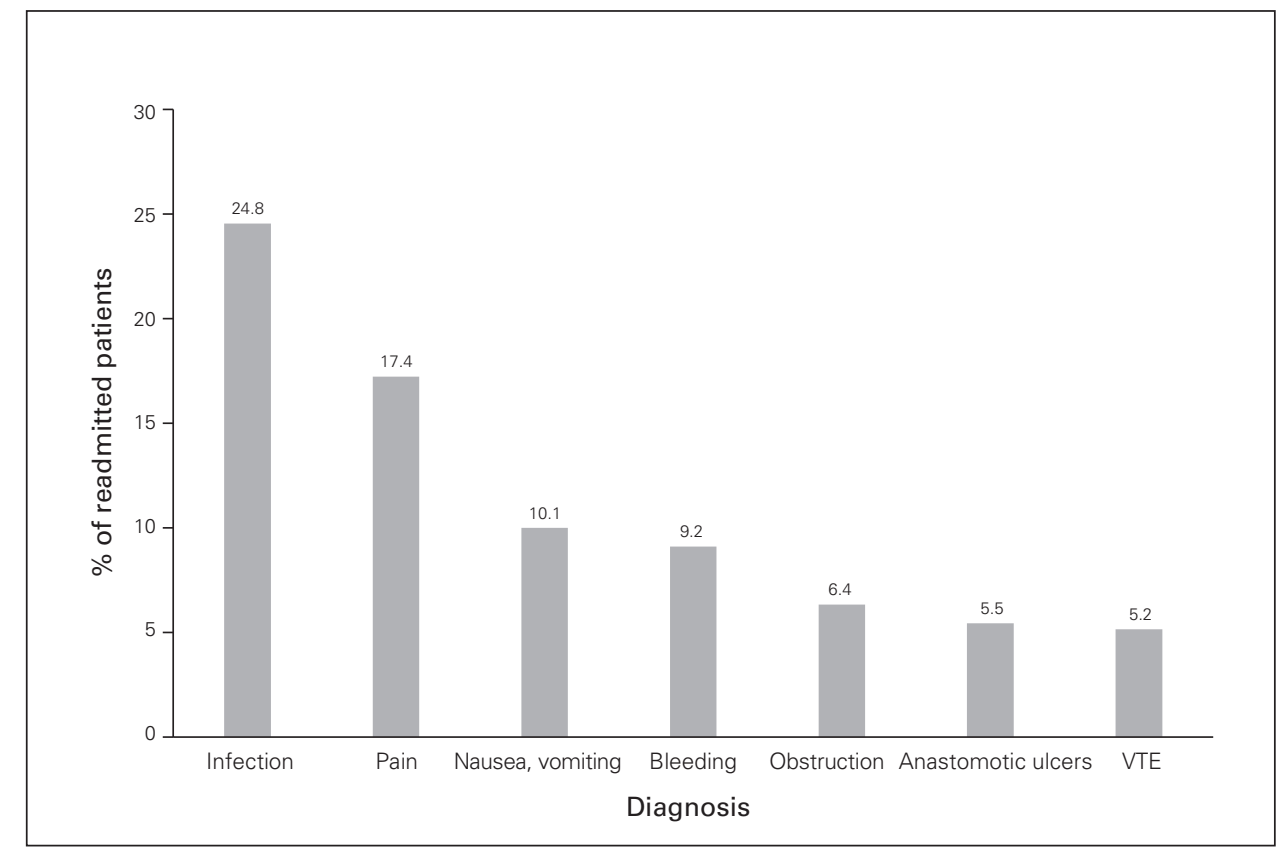

Fig. 1. Postoperative complications experienced by patients who were readmitted to hospital after bariatric surgery. VTE = venous thromboembolism. 


\section{Table 4. Perioperative factors and postoperative complications}

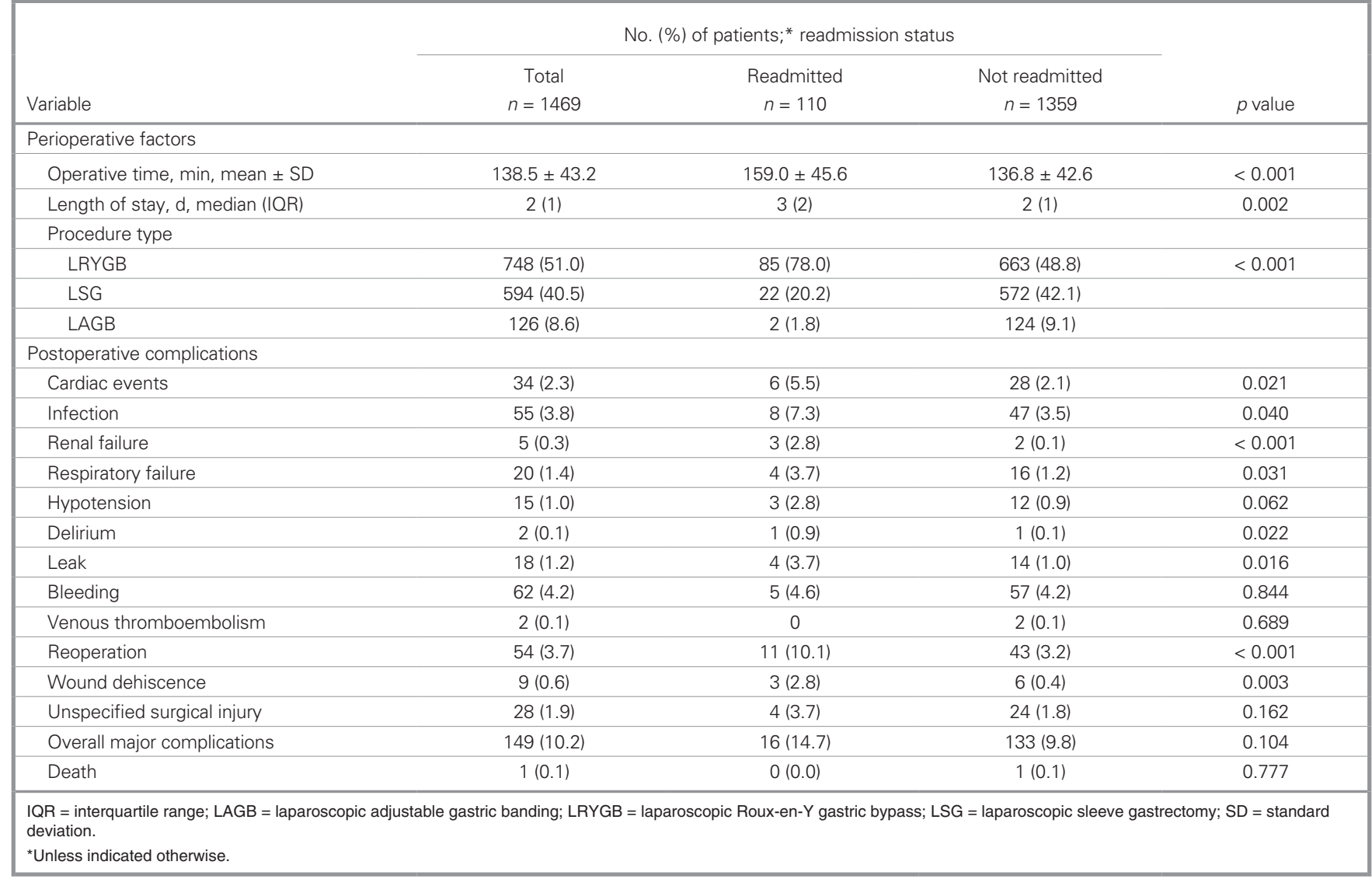

Table 5. Multivariable logistic regression analysis for factors predicting readmission

\begin{tabular}{lccc}
\hline Factor & Odds ratio $(95 \%$ Cl) & SE & $p$ value \\
\hline LRYGB v. LAGB & $5.21(1.19-22.73)$ & 3.92 & 0.028 \\
\hline LSG v. LAGB & $1.98(0.45-8.61)$ & 1.48 & 0.365 \\
\hline Operating time & $1.00(1.00-1.01)$ & 0.00 & 0.272 \\
\hline Length of stay 2-4 d (v. 1 d) & $1.28(0.81-2.02)$ & 0.30 & 0.296 \\
\hline Length of stay > 4 d (v. 1 d) & $2.18(1.03-4.63)$ & 0.84 & 0.042 \\
\hline Cardiac events & $2.01(0.74-5.45)$ & 1.02 & 0.172 \\
\hline Wound dehiscence & $2.66(0.40-17.72)$ & 2.57 & 0.313 \\
\hline Acute renal failure & $14.10(1.07-186.29)$ & 18.56 & 0.045 \\
\hline Respiratory failure & $0.63(0.13-3.06)$ & 0.51 & 0.567 \\
\hline Delirium & $0.15(0.00-10.32)$ & 0.32 & 0.379 \\
\hline Reoperation & $1.15(0.45-2.96)$ & 0.55 & 0.764 \\
\hline Unspecified surgical injury & $2.34(0.72-7.62)$ & 1.41 & 0.159 \\
\hline $\begin{array}{l}\text { Cl = confidence interval; LAGB = laparoscopic adjustable gastric banding; LRYGB = laparoscopic Roux-en-Y gastric } \\
\text { bypass; LSG = laparoscopic sleeve gastrectomy; SE = standard error. }\end{array}$ & & \\
\hline
\end{tabular}

To reduce the burden of avoidable readmissions on health care systems, other studies have looked at factors affecting readmission or perioperative complications associated with bariatric surgery. The factors reported by these studies are variable, and it can be difficult to compare results because of a lack of standardized reporting of readmission diagnoses and heterogeneity between centres and surgeon practices. In our study, length of stay during the initial hospital admission was found to be an independent predictive factor for readmission, which is consistent with other studies. ${ }^{12} \mathrm{~A}$
$8.6 \%$ of patients had this procedure. The highest readmission and complication rates were associated with LRYGB and more than half of the patients in our study underwent this procedure. ${ }^{13}$ However, when choosing between different bariatric procedures, the benefits of each procedure in terms of weight loss and obesity-related chronic diseases must be weighed against both short- and long-term risks as well as complication rates. study of data for more than 25000 patients in the Bariatric Outcomes Longitudinal Database found a 30-day readmission rate of $5.8 \%$ for LRYGB and $1.2 \%$ for LAGB with prolonged length of stay as a risk factor for readmission for both procedures. ${ }^{14}$ It is important to delineate the underlying cause of the longer initial stay. Longer initial length of stay may be due to nausea, vomiting, and fluid and electrolyte depletion, which were not 
captured by our study and not adjusted for in our multivariable model. These patients are at higher risk for readmission, as these issues may persist after discharge. Poor pain control can also prolong initial length of stay, and optimizing pain management is another way to reduce readmissions as pain was a common reason for readmission in our study. It is important to note that length of stay should be at least 1 day, as a recent analysis of the Metabolic and Bariatric Surgery Accreditation and Quality Improvement Program (MBSAQIP) database found that same-day discharge was associated with 5 -fold higher mortality than discharge on the first postoperative day after LSG.

Preventing renal failure may also reduce readmissions. In our study, acute renal failure was the single greatest independent predictor for readmission, and $60 \%$ of patients who had acute renal failure were subsequently readmitted. Renal disease appears to increase readmissions, and a recent analysis of data from the MBSAQIP database determined that a history of renal insufficiency independently predicted readmission. ${ }^{13}$ Patients who undergo bariatric surgery are at a higher risk for renal failure because of difficulty with intake from the sudden anatomic changes of bariatric surgery. Care should be taken to prevent dehydration through postoperative protocols aimed at optimizing fluid balance. Preoperative and postoperative education around appropriate postoperative oral intake may prevent dehydration as well as decrease readmissions through reductions in nausea and vomiting, which were a frequent reason for readmission. ${ }^{19}$

There may be potential to reduce readmissions by instituting very early outpatient assessments to assess for pain, nausea and infection. Our centre recently participated in the development of an enhanced recovery after surgery (ERAS) protocol with other Canadian centres. One of the interventions currently recommended by most Canadian centres is to have an early follow-up telephone call at 48 to 72 hours. This may potentially identify patients at risk for dehydration and renal failure and allow the patient to be treated early as an outpatient. Another benefit of an early follow-up call is that patient concerns can be triaged and addressed, which may also prevent returns to the emergency department.

\section{Limitations}

Our study had several limitations. The DIMR database is an administrative database, and data are coded according to ICD codes. A substantial limitation of this database is that the assignment of diagnoses and comorbidities may be subject to coding error. ICD codes also lack granularity, and it was not possible to delineate the types of infections that were the cause of readmissions. Additionally, BMI was not coded, and the impact of
BMI on remission rates could not be studied. Previous studies have shown that patients with a high BMI $\left(>53 \mathrm{~kg} / \mathrm{m}^{2}\right)$ had more severe complications and more frequent reoperations. ${ }^{20}$ However, a study using the MBSAQIP database found that BMI was not a predictor of readmission. ${ }^{13}$ Our database also did not capture clinic visits or emergency department visits, which may also increase health care costs. Although the database captures all admissions in Edmonton, it is possible that patients living outside of the Edmonton area may have been admitted to local hospitals, or the Calgary bariatric centre, potentially leading to an underestimate of true readmission rates. Furthermore, readmissions beyond the 30-day period were not evaluated in this study. This may have had a significant impact as many bariatric surgery complications, including marginal ulceration and internal herniation, tend to occur beyond 30 days. ${ }^{22}$ Despite these limitations, our study presents one of the largest analyses of readmission following bariatric surgery in a publicly funded health care system. We identified a number of variables such as hospital length of stay and acute renal failure that were independently associated with readmission. Strategies designed to reduce these complications may reduce readmission rates and their associated health care costs.

\section{Conclusion}

The overall readmission rate after bariatric surgery in our study was $7.5 \%$. Readmission was most commonly caused by potentially preventable factors such as pain, nausea or vomiting. Patients with a length of stay greater than 4 days, patients with postoperative acute renal failure and those who underwent LRYGB were at higher risk of readmission. Prevention of acute renal failure and closer follow-up of high-risk patients may reduce readmissions and health care costs.

Affiliations: From the Department of Surgery, University of Alberta, Edmonton, Alta. (Dang, Tavakoli, Switzer, Mocanu, de Gara, Birch, Karmali); and the Centre for the Advancement of Minimally Invasive Surgery, Royal Alexandra Hospital, Edmonton, Alta. (Shi, de Gara, Birch, Karmali).

Competing interests: None declared.

Contributors: J. Dang, N. Switzer, C. de Gara, D. Birch and S. Karmali designed the study. J. Dang acquired the data, which J. Dang, I. Tavakoli, V. Mocanu and X. Shi analyzed. J. Dang, I. Tavakoli and N. Switzer wrote the article, which all authors critically reviewed. All authors approved the article for publication.

\section{References}

1. World Health Organization. Obesity and overweight. Factsheet no. 311. Geneva: World Health Organization; 2012.

2. Statistics Canada. Obesity in Canadian adults, 2016 and 2017. Ottawa: Statistics Canada; 2018. Available: www150.statcan.gc.ca/n1/ pub/11-627-m/11-627-m2018033-eng.htm (accessed 2019 Aug. 9).

3. Anis AH, Zhang W, Bansback N, et al. Obesity and overweight in Canada: an updated cost-of-illness study. Obes Rev 2010;11:31-40. 
4. Cawley J, Meyerhoefer C. The medical care costs of obesity: an instrumental variables approach. F Health Econ 2012;31:219-30.

5. Sjöström L. Review of the key results from the Swedish Obese Subjects (SOS) trial - a prospective controlled intervention study of bariatric surgery. F Intern Med 2013;273:219-34.

6. Doumouras AG, Saleh F, Anvari S, et al. A longitudinal analysis of short-term costs and outcomes in a regionalized center of excellence bariatric care system. Obes Surg 2017;27:2811-7.

7. Arterburn DE, Olsen MK, Smith VA, et al. Association between bariatric surgery and long-term survival. FAMA 2015;313:62-70.

8. Buchwald H, Oien DM. Metabolic/bariatric surgery worldwide 2011. Obes Surg 2013;427-36.

9. Saleh F, Doumouras AG, Gmora S, et al. Outcomes the Ontario Bariatric Network: a cohort study. CMA7 Open 2016;4:E383-9.

10. Encinosa WE, Bernard DM, Chen C-C, et al. Healthcare utilization and outcomes after bariatric surgery. Med Care 2006;44:706-12.

11. Doumouras AG, Saleh F, Anvari S, et al. The effect of health system factors on outcomes and costs after bariatric surgery in a universal healthcare system: a national cohort study of bariatric surgery in Canada. Surg Endosc 2017;31:4816-23.

12. Doumouras AG, Saleh F, Hong D. 30-day readmission after bariatric surgery in a publicly funded regionalized center of excellence system. Surg Endosc 2016;30:2066-72.
13. Berger ER, Huffman KM, Fraker T, et al. Prevalence and risk factors for bariatric surgery readmissions. Ann Surg 2018;267:122-31.

14. Dorman RB, Miller CJ, Leslie DB, et al. Risk for hospital readmission following bariatric surgery. PLoS One 2012;7:e32506.

15. DeMaria EJ, Sugerman HJ, Meador JG, et al. High failure rate after laparoscopic adjustable silicone gastric banding for treatment of morbid obesity. Ann Surg 2001;233:809-18.

16. Khorgami Z, Andalib A, Aminian A, et al. Predictors of readmission after laparoscopic gastric bypass and sleeve gastrectomy: a comparative analysis of ACS-NSQIP database. Surg Endosc 2016;30:2342-50.

17. Celio AC, Kasten KR, Burruss MB, et al. Surgeon case volume and readmissions after laparoscopic Roux-en-Y gastric bypass: more is less. Surg Endosc 2017;31:1402-6.

18. Garg T, Rosas U, Rivas H, et al. National prevalence, causes, and risk factors for bariatric surgery readmissions. Am 7 Surg 2016;212:76-80.

19. Tayne S, Merrill CA, Shah SN, et al. Risk factors for 30-day readmissions and modifying postoperative care after gastric bypass surgery. 7 Am Coll Surg 2014;219:489-95.

20. Longitudinal Assessment of Bariatric Surgery (LABS) Consortium, Flum DR, Belle SH, et al. Perioperative safety in the longitudinal assessment of bariatric surgery. N Engl 7 Med 2009;361:445-54.

21. Zingmond DS, McGory ML, Ko CY. Hospitalization before and after gastric bypass surgery. FAMA 2005;294:1918-24. 